\title{
Correlation between attention deficit hyperactivity disorder and infrequent voiding
}

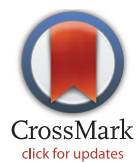

\author{
Parsa Yousefichaijan ${ }^{1}$, Ali Khosrobeigi ${ }^{2 *}$, Bahman Salehi ${ }^{1}$, Hassan Taherahmadi ${ }^{1}$, Fakhreddin Shariatmadari ${ }^{1}$, \\ Yazdan Ghandi $^{1}$, Saeed Alinejad ${ }^{1}$, Mojtaba Sharafkhah ${ }^{1}$, Simin Kianmanesh ${ }^{1}$
}

${ }^{1}$ Clinical Research Development Center of Amirkabir Hospital, School of Medicine, Arak University of Medical Sciences, Arak, Iran

${ }^{2}$ Students Research Committee, Arak University of Medical Sciences, Arak, Iran

\section{A R T I C L E I N F O}

Article Type:

Original

\section{Article History}

Received: 10 December 2017

Accepted: 1 May 2018

Published online: 13 May 2018

Keywords:

Attention deficit hyperactivity

disorder,

Child,

Infrequent voiding

\begin{abstract}
A B S T R A C T
Introduction: Attention deficit hyperactivity disorder (ADHD) can be considered as one of the widespread childhood neurological disorder, which is a well-known disorder among some chronic diseases.

Objectives: The aim of this study was to investigate ADHD in girls with infrequent voiding and compare it with healthy children.

Patients and Methods: One-hundred children aged between 5 and 12 years old with infrequent voiding and 100 healthy children without infrequent voiding, who were referred to the pediatric clinic were included in this case-control study as case and control groups, respectively, based on inclusion and exclusion criteria (Amirkabir hospital of Arak, Iran). Subjects were selected through simple random sample selection and ADHD was diagnosed by Conners' Parent Rating Scale- 48 (CPRS-48) and DSM-IV criteria and was confirmed by psychologist consult.

Results: ADHD inattentive type was observed in 6 patients in the case group and 2 patients in the control group. Moreover, 16 and 4 children, from the case group, were affected by ADHD hyperactive-impulsive type. Additionally, 7 and 4 children, from the control group, were affected by ADHD mixed type $(P=0.005)$. There were differences between the prevalence of ADHD in the case group and the control group. Data were analyzed using Binomial test in SPSS 18.

Conclusion: The result of this study showed that ADHD is more common in children with infrequent voiding.
\end{abstract}

\section{Implication for health policy/practice/research/medical education}

In a study on 100 children with infrequent voiding as the case group and 100 healthy children, ADHD is more common in children with infrequent voiding.

Please cite this paper as: Yousefichaijan P, Khosrobeigi A, Salehi B, Taherahmadi H, Shariatmadari F, Ghandi Y, et al. Correlation between attention deficit hyperactivity disorder and infrequent voiding. J Renal Inj Prev. 2018;7(3):171-174. doi: 10.15171/jrip.2018.41

\section{Introduction}

Attention deficit hyperactivity disorder (ADHD) is the main widespread disorder in childhood which is clinically manifested as a neurobehavioral disorder. Three types of DSM-V (statistical manual of mental disorders, fifth in edition) have been classified in ADHD inattentive type, ADHD hyperactive-impulsive type, and ADHD mixed type, according to DSM-V criteria (1-3).

The studies, conducted on the children at school age, have revealed ADHD in $10 \%-50 \%$ of them. It is not wellunderstood that what factors can arise ADHD in children.
Evidence recognizes underlying genetic defects and CNS dysfunction as the main causes (4-6).

Additionally, some studies showed a meaningful link between ADHD and different types of chronic diseases or mental disorders like depression, behavioral, emotional status, language as well as hearing disorders. Even some neurological disorders like epilepsy and abnormal electroencephalogram (EEG) can be related to ADHD in children. As the evidence suggests, urinary disorders can be also associated with ADHD in children (7-9). Infrequent voiding is a common problem of urination that 
mostly has an association with urinary tract infections. These children void only twice daily (normal voiding is 4-7 times per a day). This disorder is a behavioral disorder (10).

The most effective treatment in both of them (ADHD and infrequent voiding) is low-dose methylphenidate. In addition, infrequent voiding can be associated with behavioral problems in children (1-3).

The importance and prevalence of ADHD and voiding disorders such as infrequent voiding in children suggest a relationship between these two disorders.

\section{Objectives}

The aim of this study was to investigate ADHD in children with infrequent voiding and compare it with healthy children.

\section{Patients and Methods Study population}

This case-control study was performed on 200 children aged 5-12 years old who were referred to the pediatric clinic of Amir-Kabir hospital in Arak, Iran. Of the 200 children under study, 100 children with infrequent voiding as the case group, and 100 healthy children without infrequent voiding as the control group were included in the study. Subjects were selected by simple random sampling method. Infrequent voiding was diagnosed by a pediatric nephrologist, based on the history of voiding. Clinical interviews were carried out with the children and their parents to study the inclusion/exclusion criteria. Inclusion criteria were children aged 5-12 years old with infrequent voiding according to diagnostic criteria written in consent from patients' parents or guardians.

Exclusion criteria were 1) History of major depressive disorder (MDD), anxiety disorders (ADs), and other considerable psychiatric disorders or nervous system disorders. 2) History of considerable or chronic medical disorders such as epilepsy, asthma, diabetes and immune deficiency, malignancy.

According to the diagnostic workup of infrequent voiding and its definition, the children with infrequent voiding were entered to the study as the case and control groups which were selected from children who had referred to hospital as an outpatient. After primary evaluation regarding exclusion/inclusion criteria and obtaining the informed consent from children's parents for participating in the study, basic information (age and gender) was recorded. ADHD was defined according to DSM-IV criteria as the presence of disease symptoms for at least six months continuously and in two separate environments (both at home and at school) without any organic causes. It was diagnosed by Conner's Parent Rating Scale - 48 (CPRS-48). After CPRS-48 was completed by the parents and the children identified with any form of ADHD, the children were referred to an expert psychiatrist as the project administrator to confirm ADHD diagnosis by clinical interview and based on DSM-IV diagnostic criteria. Conner's Parent Rating Scale (CPRS) was standardized by Conner et al, in 1999. It has two versions of 93-item and 48-item. The present research used the 48 -item version. This version of Conner's Questionnaire evaluates five factors of conduct, psychosomaticimpulsivity, hyperactivity, anxiety and learning problems and also has four choices scored from 0 (never) to 3 (very high). The score of each article is converted into $t$ scores with the average of 50 and standard deviation of 10 . When the $t$ scores of 12 standard deviations are higher than the average, the individual has a problem. The sensitivity and specificity of this form of Conner Questionnaire for the diagnosis of children with ADHD were $90.3 \%$ and $81.2 \%$, respectively.

\section{Ethical issues}

1) The research followed the tenets of the Declaration of Helsinki; 2) Informed consent was obtained, and they were free to leave the study at any time. 3) The research was approved by the ethical committee of Arak University of Medical Sciences. The project was approved in July 2013 (ethical code \# 937).

\section{Statistical analysis}

The collected data were analyzed using SPSS version 18.0 (SPSS Inc, Chicago, Ill, USA) and descriptive statistics methods for frequency determination. Moreover, student $t$ test and chi-square test were used for data analysis. $P$ values less than 0.05 were considered significant.

\section{Results}

The mean age of children in the case and control groups was calculated at $7.80 \pm 2.54$ years and $9.29 \pm 1.88$ years, respectively $(P=0.007)$. Of the 100 children in the case group, 46 children were girls and 54 children were boys and of the 100 children in control group, 41 children were girls and 59 children were boys $(P=0.567)$ (Table 1$)$. Of $200(100 \%)$ children under study in both groups, 8 (4\%), $20(10 \%)$ and $11(5.5 \%)$ children were affected by ADHD inattentive type, ADHD hyperactive-impulsive type, and ADHD mixed type, respectively. ADHD inattentive type was observed in 6 patients in the case group and 2 patients in the control group. Moreover, 16 and 4 children, from the case group, were affected by ADHD hyperactiveimpulsive type, and 7 and 4 children from the control group were affected by ADHD mixed type $(P=0.005)$ (Table 2). There were differences between the prevalence of $\mathrm{ADHD}$ in the case group and the control group.

Table 1. Characteristics of children

\begin{tabular}{lllll}
\hline Parameters & & Case & Control & P value \\
\hline \multirow{2}{*}{ Gender } & Girl, No. (\%) & $46(46)$ & $41(41)$ & \\
& Boy, No. (\%) & $54(54)$ & $59(59)$ & 0.567 \\
Age (y) & Mean \pm SD & $7.80 \pm 2.54$ & $9.29 \pm 1.88$ & 0.007 \\
\hline
\end{tabular}


Table 2. Attention deficit hyperactivity disorder types in children

\begin{tabular}{lcc}
\hline ADHD type & Case & Control \\
\hline Inattentive type, No. (\%) & $6(6)$ & $2(2)$ \\
Hyperactive-impulsive type, No. (\%) & $16(16)$ & $4(4)$ \\
Mixed type, No. (\%) & $7(7)$ & $4(4)$ \\
None of them, No. (\%) & $71(71)$ & $90(90)$ \\
Total, No. (\%) & $100(100)$ & $100(100)$ \\
\hline
\end{tabular}

\section{Discussion}

As widely accepted for ages, it revealed that the infrequent voiding could be developed because of inciting psychological events or psychopathology. However, there is a great uncertainty reported in recent studies on whether these disorders can associate with social stress factors or psychopathology. Our study showed a significant relationship between disorders including ADHD inattentive type, ADHD hyperactive-impulsive type, and ADHD mixed type - and infrequent voiding in children. We found that the prevalence of ADHD in the children with infrequent voiding was exceeded compared to the control group. According to some authors' studies in this field and the results of our study, the hypothesis about the relationship between ADHD and infrequent voiding is not rejected, however, further studies are recommended due to lack of studies in this field.

\section{Conclusion}

There were differences between the prevalence of ADHD in the children with infrequent voiding and the control group. However, due to the importance of relationships between different types of psychiatric disorders such as $\mathrm{ADHD}$ and infrequent voiding and lack of enough evidence concerning the relationship between these two disorders, conducting further studies in this field is recommended.

We would suggest that future studies should target the change in current treatment protocols which may be organizing a therapeutic team by adding a child psychologist for treating the patient with infrequent voiding. The psychologist could add to the therapeutic endeavors by helping to investigate the parent-child relationship and develop a specific behavioral plan that could possibly improve the clinical outcome.

\section{Limitations of the study}

Some of parents did not cooperate in completing the questionnaires. Additionally due to the disagreement of some parents with filling up the ADHD questionnaire, and lack of cooperation of the children with psychiatric examination by a psychiatrist, our researchers had some limitations. Though this criterion caused to exclude some children eligible for the study, we attempted to remove such limitation by encouraging the parents for participating in the study and help them in fill the questionnaire. Nevertheless, all findings of our study lead us to a reasonable suppose that a central neurochemical dysfunction may be shared between infrequent voiding and ADHD. Obtained results from the last decade studies conducted on treatment of urinary incontinence in children with ADHD, causes an impression that the researchers failed to provide more successful treatment of these patients.

\section{Acknowledgements}

The research team wishes to thank vice-chancellor of research for the financial support and also the children and their parents contributing to this research. The project was approved in July 2013 (ethical code \# 937).

\section{Authors' contribution}

PY; Study design, preparation of manuscript, final revision, and data interpretation. AK; Study design, manuscript edition, and final revision. SK, BS, HT, FS, YG, SA and MS; Study design, data gathering and data interpretation.

\section{Conflicts of interest}

The authors declared no competing interests.

\section{Ethical considerations}

Ethical issues (including plagiarism, data fabrication, double publication) have been completely observed by the authors.

\section{Funding/Support}

This research project was approved by office of Vice Chancellor for Research and the number of this project is 937. This project started in July 2013 and finished in September 2014.

\section{References}

1. Yousefichaijan P, Sharafkhah $M$, Rafiei M, Salehi B. Attention-deficit/hyperactivity disorder in children with overactive bladder; a case-control study. J Renal Inj Prev. 2016;5:193-9. doi: 10.15171/jrip.2016.41

2. Yousefichaijan P, Sharafkhah M, Vazirian S, Seyedzadeh A, Rafeie M, et al. Attention-Deficit/Hyperactivity Disorder in Children Undergoing Peritoneal Dialysis, Nephro-Urol Mon. 2015;7:e24427. doi: 10.5812/numonthly.24427.

3. Yousefi Chaijan P, Sharafkhah M, Salehi B, Rafiei M. Attention deficit hyperactivity disorder in children with early stages of chronic kidney disease. Med J Islam Repub Iran. 2015;29:174-180.

4. Yousefi P, Salehi B, Rafeie M, Firouzifar M, Mousavinejad S. Parents function and behavioral disorders in children with and without diurnal voiding dysfunction: a comparative study. Zahedan Journal of Research in Medical Sciences. 2014;16:1-4.

5. Yousefi P, Firouzifar MR, Cyrus A. Correlation between sacral ratio and primary enuresis. J Nephropathol. 2012;1: 183-7. doi: 10.5812/nephropathol.8120. 
6. Yousefichaijan P, Sharafkhah M, Salehi B, Rafiei M. Attention deficit hyperactivity disorder in children with primary monosymptomatic nocturnal enuresis: A casecontrol study. Saudi J Kidney Dis Transpl. 2016;27:73-80. doi: $\quad 10.4103 / 1319-2442.174077$.

7. Yousefichaijan P, Khosrobeigi A, Salehi B, Taherahmadi H, Shariatmadari F, Ghandi Y, et al. Incidence of obsessivecompulsive disorder in children with nonmonosymptomatic primary nocturnal enuresis. J Pediatr Neurosci. 2016;11:197-9. doi: 10.4103/1817-1745.193371.

8. Yousefichaijan P, Salehi B, Rafiei M, Ghadimi N,
Taherahmadi H, Hashemi SM, Naziri M. Emotional disorders in children with monosymptomatic primary nocturnal enuresis. J Pediatr Nephrol. 2015;3:22-5.

9. Yousefichaijan P, Salehi B, Khosrobeigi A, Hajirahimi M, Rafiei M, Taherahmadi H, et al. Pollakiuria and its relationship with obsessive compulsive disorder. J Renal Inj Prev. 2018;7:35-37. doi: 10.15171/jrip.2018.08.

10. Elder JS. Enuresis and Voiding Dysfunction. In: Behrman RE, ed. Nelson Textbook of Pediatrics. 20th ed. Philadelphia: Elsevier; 2016. p. 2581-6.

Copyright $\odot 2018$ The Author(s); Published by Nickan Research Institute. This is an open-access article distributed under the terms of the Creative Commons Attribution License (http://creativecommons.org/licenses/by/4.0), which permits unrestricted use, distribution, and reproduction in any medium, provided the original work is properly cited. 